\title{
Solution-Processed Nanocomposites Containing Molybdenum Oxide and Gold Nanoparticles as Anode Buffer Layers in Plasmonic- Enhanced Organic Photovoltaic Devices
}

\author{
Kim-Shih Tan, ${ }^{\dagger}$ Ming-Kai Chuang, ${ }^{\dagger}$ Fang-Chung Chen, ${ }^{*}{ }^{\dagger}$ and Chain-Shu Hsu ${ }^{\ddagger}$
}

${ }^{\dagger}$ Department of Photonics and Display Institute, National Chiao Tung University, Hsinchu 30010, Taiwan

${ }^{\ddagger}$ Department of Applied Chemistry, National Chiao Tung University, Hsinchu 30010, Taiwan

Supporting Information

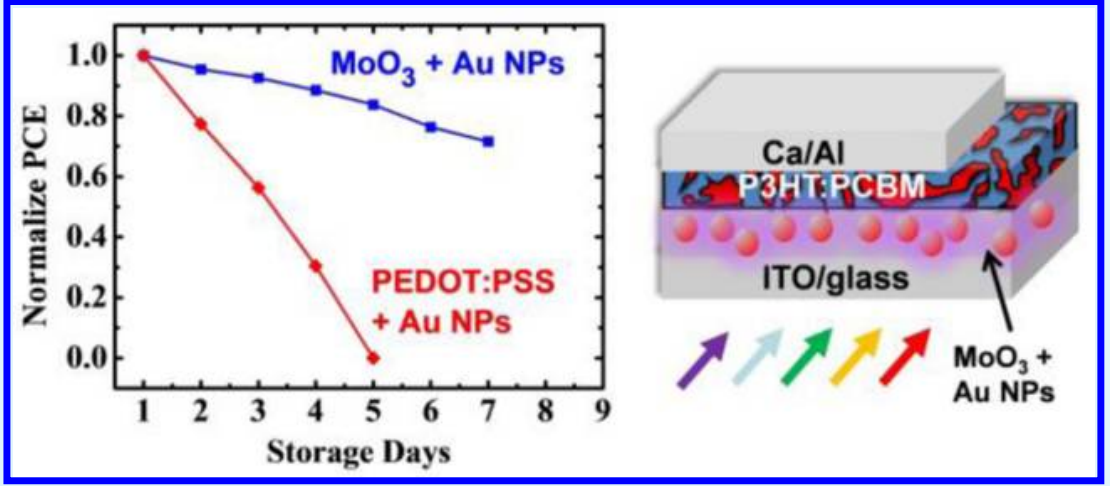

ABSTRACT: Solution-processed nanocomposites containing molybdenum oxide $\left(\mathrm{MoO}_{3}\right)$ and gold nanoparticles (Au NPs) have been used as anode buffer layers in organic photovoltaic devices (OPVs). The resulting devices exhibit a remarkable enhancement in power conversion efficiency after Au NPs were incorporated into the device. Such enhancements can be attributed to the localized surface plasmon resonance induced by the metallic nanostructures. We have also found that the rate of exciton generation and the probability of exciton dissociation were increased. Furthermore, the devices made of the $\mathrm{MoO}_{3}$ buffer layer containing Au NPs exhibited superior stability. This work opens up the possibility of fabricating OPVs with both high efficiency and a prolonged lifetime.

KEYWORDS: molybdenum oxide, gold nanoparticles, plasmonic, polymer, photovoltaic devices, stability

\section{INTRODUCTION}

Organic photovoltaic devices (OPVs) have been extensively studied because they have many advantageous properties, including low-cost fabrication, light weight, mechanical flexibility, and an amazing short period of energy pay-back time, compared to photovoltaic cells based on inorganic semiconductors. ${ }^{1-4}$ Over the past few years, the efficiencies of OPVs have been improved substantially to over $10 \%{ }^{4}$ Nevertheless, power conversion efficiency (PCE) of these devices still needs to be improved to compete with inorganic photovoltaic devices. Additionally, inferior operating stability is also a problem requiring emphatic concern.

Organic semiconductors usually exhibit low charge-carrier mobility and short exciton diffusion length. As a result, there exists a trade-off between light absorption and charge-collection efficiency in OPVs. ${ }^{5,6}$ The thickness of the photoactive layer is limited largely to $\sim 100 \mathrm{~nm}$ for effective charge collection and reduced charge-recombination loss. To increase the absorption efficiency using thin films, many light-trapping approaches have been proposed. ${ }^{5}$ For example, the introduction of a surface plasmon effect induced by metallic nanostructures ${ }^{7}$ and the incorporation of optical spacers for redistribution of the electrical field in the device ${ }^{8,9}$ have been demonstrated successfully. Between these approaches, light trapping on the basis of localized surface plasmon resonance (LSPR) effects has received much attention as a means of improving the absorption efficiency., ${ }^{70-17}$ Specifically, metal nanoparticles (NPs) are often introduced into the devices for triggering LSPRs. Their unique optical properties, including strong light scattering and local field enhancement, improve the absorption efficiency of OPVs substantially.

One simple method for incorporating the metal NPs into the devices is through doping soluble NPs in the anode buffer layers, such as poly(3,4-ethylenedioxythiophene):poly(styrenesulfonate) (PEDOT:PSS). ${ }^{12,13}$ The use of PEDOT:PSS, however, usually suffers from the problem of long-term stability because of its acidic and hygroscopic nature. ${ }^{18}$ Alternatively, transition-metal oxides, such as molybdenum oxide $\left(\mathrm{MoO}_{3}\right)$

Received: August 13, 2013

Accepted: November 15, 2013

Published: November 15, 2013 
and vanadium oxide $\left(\mathrm{V}_{2} \mathrm{O}_{5}\right)$, can be used as an effective anode buffer layer for enhancing the charge-collection efficiency. ${ }^{19-22}$ These metal oxides are usually fabricated through vacuum processes, which require higher cost. More recently, a simple, solution-processable method for synthesizing $\mathrm{MoO}_{3}$ buffers has been reported using ammonium heptamolybdate $\left(\mathrm{NH}_{4}\right)_{6} \mathrm{Mo}_{7} \mathrm{O}_{24}-4 \mathrm{H}_{2} \mathrm{O}$ as a precursor. ${ }^{22}$ The precursor was decomposed to $\mathrm{MoO}_{3}$, resulting in an excellent buffer with high device performance comparable to that using PEDOT:PSS. Herein, we report one simple approach for preparing gold nanoparticles (AuNPs) $/ \mathrm{MoO}_{3}$ composites as the anode buffer layers of OPVs. The embedded Au NPs induced LSPR, which enhanced the local electromagnetic field of the active layer, thereby improving the device performance. The $\mathrm{AuNP} / \mathrm{MoO}_{3}$ nanocomposite layer is one of the most promising candidates for increasing the light-harvesting ability without significantly sacrificing the charge transport and/or collection efficiencies in OPVs. More importantly, the devices made of the $\mathrm{MoO}_{3}$ buffer layer containing Au NPs exhibited superior stability. This work opens up the possibility of fabricating OPVs with both high efficiency and prolonged lifetime.

\section{MATERIALS AND METHODS}

2.1. Preparation of $\mathrm{MoO}_{3}$ and $\mathrm{AuNP} / \mathrm{MoO}_{3}$ Thin Films. To prepare the $\mathrm{MoO}_{3}$ solutions, ammonium heptamolybdate was dissolved in deionized water to form a $0.2 \mathrm{wt} \%$ solution. $^{22}$ The $\mathrm{pH}$ of the solution was adjusted to between 1 and 1.5. Then, the precursor solution was heated at $80{ }^{\circ} \mathrm{C}$ for $2 \mathrm{~h}$ in air. Ammonium heptamolybdate was decomposed to three components: $\mathrm{MoO}_{3}$, $\mathrm{NH}_{3}$, and $\mathrm{H}_{2} \mathrm{O}$. ${ }^{22}$ Gaseous $\mathrm{NH}_{3}$ was easily evaporated under the experimental conditions, and $\mathrm{MoO}_{3}$ was left as the only solute in the solution. The $\mathrm{Au}$ NPs were synthesized following a procedure developed in a previous work. ${ }^{23}$ Figure $1 \mathrm{~b}$ displays the scanning

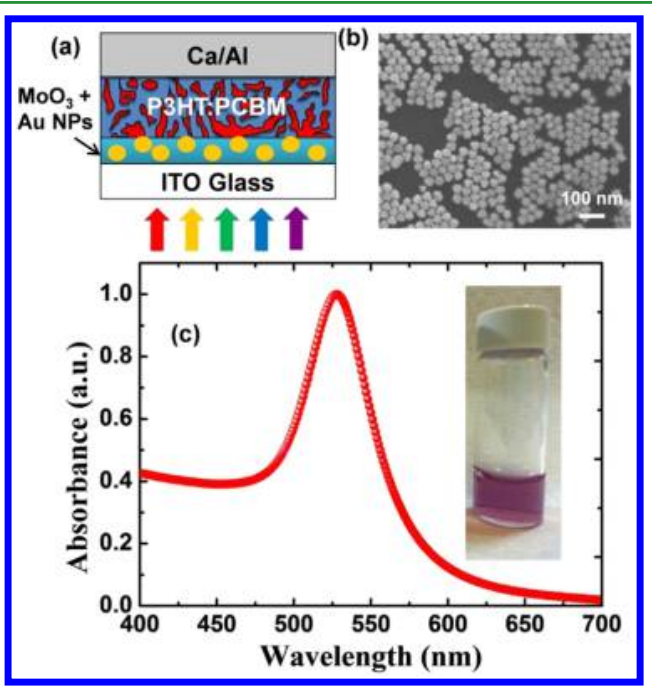

Figure 1. (a) Device structure of the OPVs in this study. (b) SEM image of the Au NPs. The average size was $40 \pm 5 \mathrm{~nm}$. (c) UV-vis absorption spectrum of the Au NPs suspended in water. The inset shows a photograph of the Au NP solution.

electron microscopy (SEM) image of the octahedral Au NPs. We observed that the average particle size of the Au NPs was approximately $40 \mathrm{~nm} .{ }^{17}$ For the preparation of the composite buffer layers, the Au NPs were mixed with the as-prepared $\mathrm{MoO}_{3}$ solution (Figure 2a). The anode buffer layers were prepared by spin coating the aqueous mixtures containing both $\mathrm{Au}$ NPs and $\mathrm{MoO}_{3}$ precursors onto the $\mathrm{UV}-\mathrm{O}_{3}$-pretreated indium tin oxide (ITO)-coated glass substrates. The samples were annealed at $150{ }^{\circ} \mathrm{C}$ for $10 \mathrm{~min}$ in air. Figure $2 \mathrm{~b}$

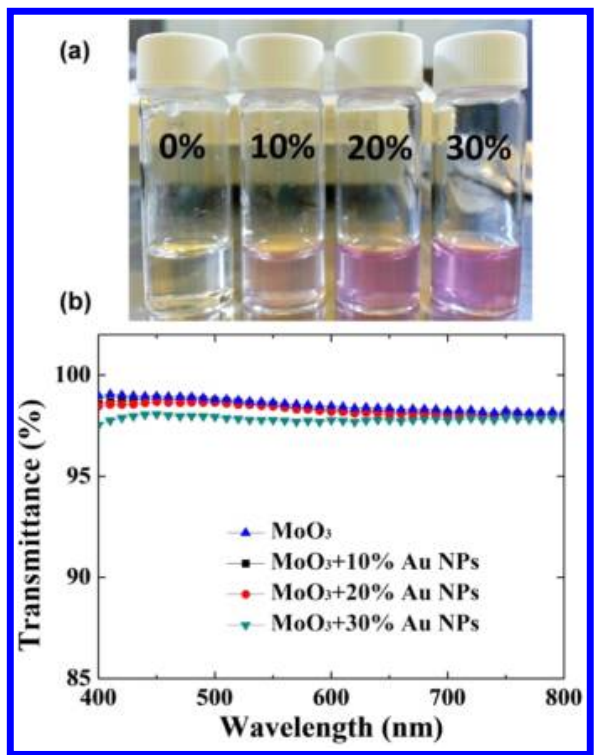

Figure 2. (a) Photograph of the $\mathrm{MoO}_{3}$ solution containing various volume ratios of $\mathrm{Au}$ NPs for preparing the anode buffer layers. (b) Transmittance spectra of the $\mathrm{MoO}_{3}$ thin films deposited on ITO glass substrates. Different concentrations of Au NPs were embedded in the thin films.

reveals the transmittance spectra of the $\mathrm{MoO}_{3}$ solution incorporating $\mathrm{Au}$ NPs with different concentrations. We can see that the neat $\mathrm{MoO}_{3}$ film exhibited high transmittance in the visible spectral region. Because the plasmon band of Au NPs was around $530 \mathrm{~nm}$, the transmittance decreased upon the addition of Au NPs.

2.2. Fabrication of Photovoltaic Devices. The devices were fabricated on ITO-coated glass substrates. The device structure in this work is illustrated in Figure 1a. The ITO substrates were treated with $\mathrm{UV}$-ozone prior to use. The $\mathrm{Au} \mathrm{NP} / \mathrm{MoO}_{3}$ films were prepared using spin coating as described above. The photoactive layer comprising regioregular poly(3-hexylthiophene) (P3HT) and 1-(3methoxycarbonyl)propyl-1-pheny[6,6]methanofullerene (PCBM) at a weight ratio of 1:1 was spin-coated from a solution of 1,2dicholorobenzene on the anode buffer layers. After solvent annealing, ${ }^{24,25}$ the dried film was further annealed at $110{ }^{\circ} \mathrm{C}$ for 10 min. The resulting thickness of the active layer was $\sim 180 \mathrm{~nm}$. Finally, the metals $\mathrm{Ca}(30 \mathrm{~nm})$ and $\mathrm{Al}(100 \mathrm{~nm})$ were thermally evaporated sequentially to form the cathodes. The device active area was $0.1 \mathrm{~cm}^{2}$. The thickness of the $\mathrm{MoO}_{3}$ buffer layers was ca. $10 \mathrm{~nm}$.

2.3. Characterization. For the device characteristics, a Keithley 2400 source measure unit was used for recording the current densityvoltage $(J-V)$ curves. The electrical properties of the devices under illumination conditions were measured using a $150 \mathrm{~W}$ Thermal Oriel solar simulator equipped with standard AM 1.5G filters. The light intensity of the simulator was further calibrated using a $\mathrm{Si}$ photodetector containing a KG5 filter. ${ }^{26}$ The incident photon-toelectron conversion efficiency (IPCE) curves were obtained from a measurement system (Enli Technology) composed of a quartztungsten-halogen lamp, a monochromator, an optical chopper, a lockin amplifier, and a calibrated silicon-based diode.

\section{RESULTS AND DISCUSSION}

Figure 3 displays the $J-V$ characteristics of the devices fabricated with various amounts of Au NPs; the measurements were performed under standard illumination conditions (AM $1.5 \mathrm{G}, 100 \mathrm{~mW} \mathrm{~cm}^{-2}$ ). The controlled device, in which a layer of pristine $\mathrm{MoO}_{3}$ was used as the anode buffer, exhibited an open-circuit voltage $\left(V_{\text {oc }}\right)$ of $0.60 \mathrm{~V}$, a short-circuit current $\left(J_{\mathrm{sc}}\right)$ of $10.2 \mathrm{~mA} \mathrm{~cm}^{-2}$, and a fill factor $(F F)$ of 0.60 . The calculated PCE was $3.68 \%$. The results were consistent with those 


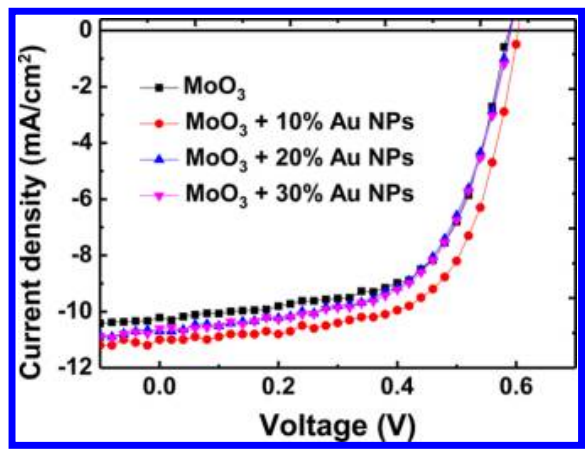

Figure 3. $J-V$ characteristics obtained under AM 1.5G illumination $\left(100 \mathrm{~mW} \mathrm{~cm}{ }^{-2}\right.$ ) of the OPVs prepared with $\mathrm{MoO}_{3}$ layers incorporating various amounts of Au NPs.

reported previously. ${ }^{22}$ After the incorporation of Au NPs to the $\mathrm{MoO}_{3}$ buffer layer, the photocurrent and $F F$ were improved. For the optimized device, the $J_{\text {sc }}$ and FF values were improved to $10.9 \mathrm{~mA} \mathrm{~cm}^{-2}$ and 0.65 , respectively. The values of $V_{\mathrm{oc}}$ remained unchanged $(0.60 \mathrm{~V})$, indicating that the anode interface was not significantly affected by the Au NPs. As a result, the PCE was increased to $4.20 \%$. We also extracted the device series resistance $\left(R_{\mathrm{s}}\right)$ from the $J-V$ curves measured in the dark (Figure S1). The values of $R_{\mathrm{s}}$ increased from 1.7 to 1.9 $\Omega \mathrm{cm}^{2}$ after the addition of $10 \%$ Au NPs, suggesting that the device improvement was not due to the reduction in device resistance. Rather, the advantageous LSPR effect induced by the $\mathrm{Au}$ NPs overwhelmed the inferior electrical properties of the devices containing $\mathrm{Au} \mathrm{NPs}{ }^{17} \mathrm{We}$ also note that a further increase in the amount of the Au NPs resulted in a decrease in the value of $J_{\text {sc }}$. We suspected that the excess Au NPs might lead to enhanced backward scattering. Furthermore, the high concentration of NPs might also increase the resistivity of the buffer layers. From the $J-V$ curves measured in the dark, we indeed obtained an increased $R_{\mathrm{s}}$ to $2.4 \Omega \mathrm{cm}^{2}$ after the addition of $30 \%$ Au NPs. Table 1 summarizes the device performance in this work.

Figure 4a shows the IPCE spectra of the solar cells prepared with different anode buffers. We can see that the trends in the IPCE were consistent with those for the values of $J_{\mathrm{sc}}$. The devices containing $\mathrm{Au}$ NPs exhibited higher efficiencies. Furthermore, we also noted that the spectral shape changed after the use of $\mathrm{Au}$ NP solutions. The efficiencies increased substantially within the spectral range from 450 to $600 \mathrm{~nm}$. The peak efficiency was improved to $66 \%$ at $550 \mathrm{~nm}$ for the device containing $10 \% \mathrm{Au}$ NPs. Figure $4 \mathrm{~b}$ displays the corresponding curve of the increase in IPCE ( $\triangle \mathrm{IPCE})$ for the solar cell containing 10\% Au NPs; the extinction spectrum of the Au NPs is also included in Figure $4 \mathrm{~b}$ for comparison. The spectral regime in which the IPCE values were increased was matched with the plasmonic band of the $\mathrm{Au}$ NPs. The spectral

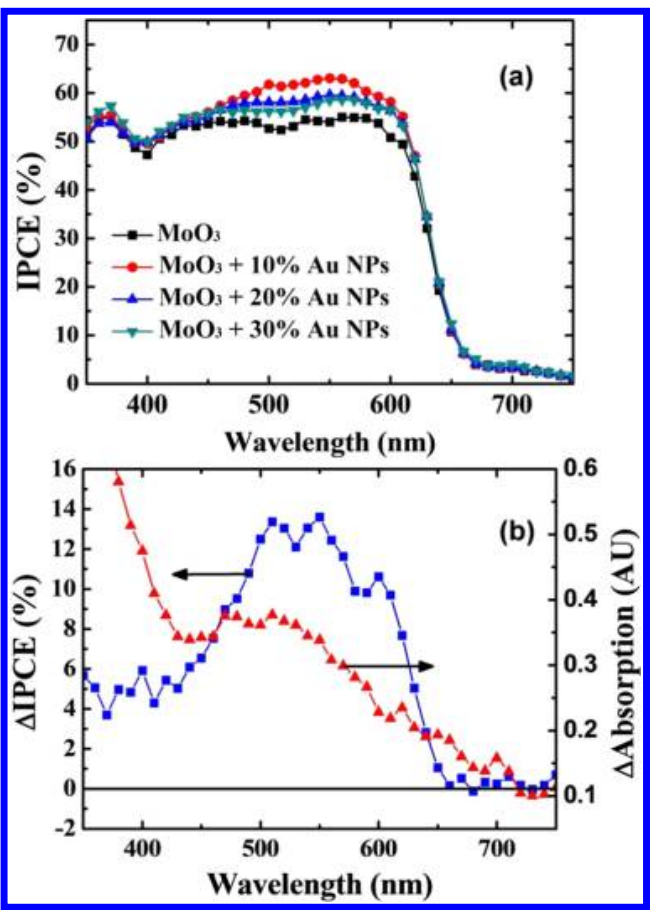

Figure 4. (a) IPCE spectra for the photovoltaic devices prepared with various concentrations of $\mathrm{Au} \mathrm{NP}$ solutions. (b) Increase in IPCE ( $\triangle \mathrm{IPCE}$ ) after the use $10 \%$ Au NPs in the anode buffer layer and the absorption spectrum of the Au NPs. The absorption spectrum was obtained using a neat $\mathrm{MoO}_{3}$ thin film as the baseline.

consistence suggested that the LSPR contributed to the device enhancement.

The morphology changes of the $\mathrm{MoO}_{3}: \mathrm{Au}$ NP layer were investigated using atomic force microscopy (AFM), and the topographic images are displayed in Figure 5. The root-meansquare (rms) roughness of the $\mathrm{MoO}_{3}$ film was $8.8 \mathrm{~nm}$ (Figure 5a). Upon the addition of $\mathrm{Au}$ NPs, we observed obvious changes in the surface morphology of the $\mathrm{MoO}_{3}$ films. The rms roughness was increased to $10.4,11.9$, and $12.7 \mathrm{~nm}$ after the incorporation of 10,20 , and $30 \%$ of $\mathrm{Au}$ NP solutions, respectively. The rough surface might induce trap states at the interfaces and somehow affect the charge-transport/ collection efficiencies of the OPVs, resulting in a lower photocurrent. The increased $R_{\mathrm{s}}$ value of the devices containing 20 and $30 \%$ Au NPs further confirmed this assumption. For the device containing $10 \% \mathrm{Au}$ NPs, however, the drawback of the poor interfaces was overwhelmed by the higher degree of absorption upon the addition of Au NPs, thereby improving the overall device PCE.

The transmission electron microscopy (TEM) image of the $\mathrm{MoO}_{3}$ containing $10 \% \mathrm{Au}$ NP solution was obtained to study further the morphology of the thin films (Figure S2). We observed large particles, which might be due to the formation of $\mathrm{MoO}_{3}$. However, the dark dots should be Au NPs in the

Table 1. Electrical Characteristics of Devices Incorporating Various Amounts of Au NPs

$\begin{array}{ccccc}\text { concentration of Au NPs (\%) } & V_{\mathrm{oc}}(\mathrm{V}) & J_{\mathrm{sc}}\left(\mathrm{mA} \mathrm{cm}^{-2}\right) & \text { PF } & R_{\mathrm{s}}(\Omega \mathrm{cm})^{a} \\ 0 & 0.60 \pm 0.01 & 10.2 \pm 0.17 & 0.60 \pm 0.02 & 3.68 \pm 0.08 \\ 10 & 0.60 \pm 0.01 & 10.9 \pm 0.20 & 0.64 \pm 0.02 & 4.20 \pm 0.11 \\ 20 & 0.60 \pm 0.01 & 10.7 \pm 0.25 & 0.62 \pm 0.03 & 3.97 \pm 0.12 \\ 30 & 0.60 \pm 0.01 & 10.6 \pm 0.21 & 0.61 \pm 0.02 & 3.1\end{array}$

${ }^{a}$ Device series resistance $\left(R_{\mathrm{s}}\right)$ was obtained from the inverse slope of the dark $J-V$ curve. 


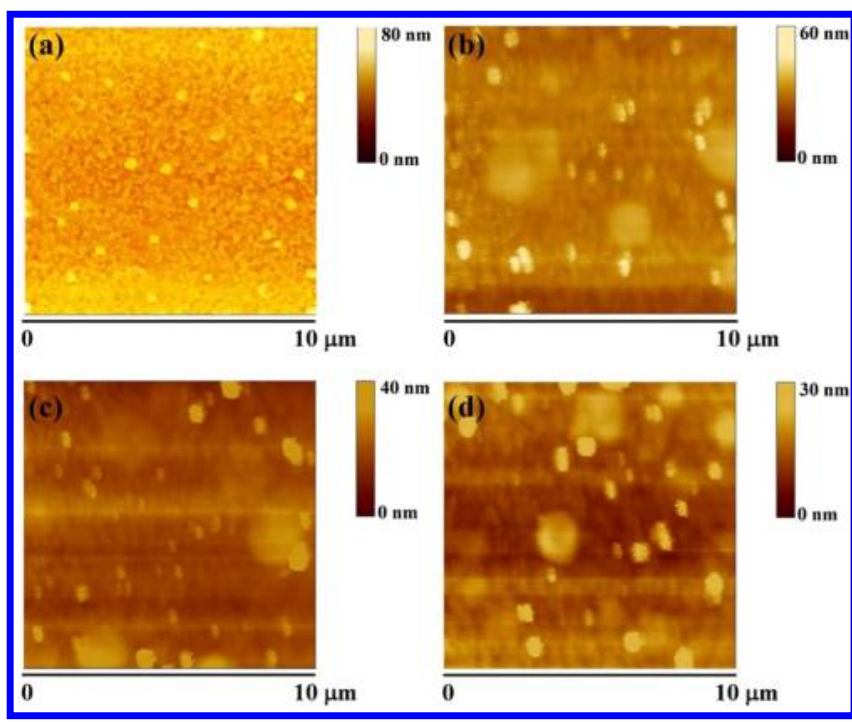

Figure 5. AFM images $\left(10 \times 10 \mu \mathrm{m}^{2}\right)$ of the $\mathrm{MoO}_{3}$ buffer layers prepared with different concentrations of Au NP solutions: (a) 0, (b) 10, (c) 20, and (d) $30 \%$ Au NP solution doping.

nanocopposites, which did not aggregate in the thin films. The results suggest that the rougher surface, as revealed in the AFM images, could be due to the aggregation of $\mathrm{MoO}_{3}$ particles.

The photocurrent behavior of the plasmonic-enhanced device was further investigated to study the LSPR effects on the device performance. Through the calculation of the maximum generation rate $\left(G_{\max }\right)$, we were able to evaluate the overall light harvesting. The devices were biased swept from +1 to $-10 \mathrm{~V}$ under $100 \mathrm{~mW} \mathrm{~cm} \mathrm{~cm}^{-2}$ illumination (AM 1.5G). Figure 6a displays the dependence of the photocurrent $\left(J_{\mathrm{ph}}\right)$ on the effective applied voltage $\left(V_{\text {eff }}\right)$. The photocurrent accounts for the dark current $\left(J_{\mathrm{D}}\right)$ using the equation ${ }^{27,28}$

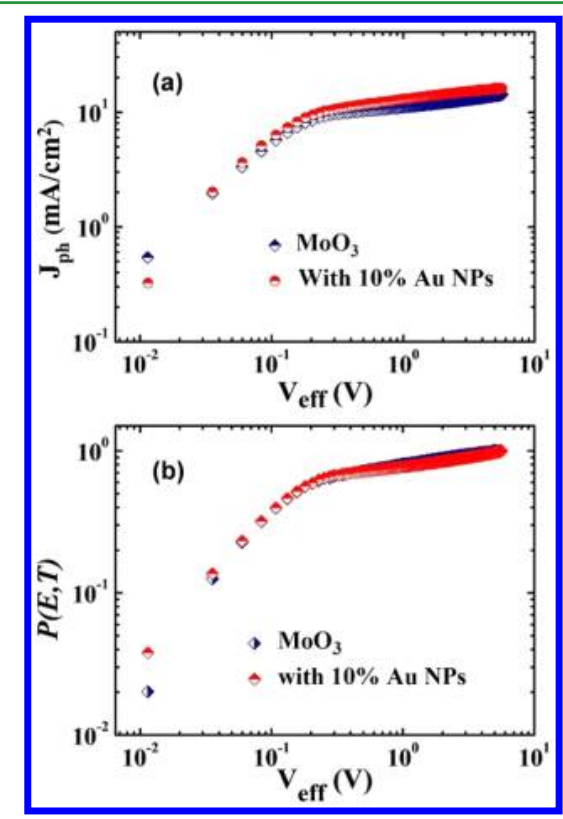

Figure 6. (a) Plots of photocurrent density as a function of the effective bias for devices prepared with neat $\mathrm{MoO}_{3}$ and $\mathrm{MoO}_{3}$ containing 10\% Au NP solution. (b) Exciton dissociation probability $[P(E, T)]$ plotted with respect to $V_{\text {eff }}$ for the device prepared with and without Au NPs.

$$
J_{\mathrm{ph}}=J_{\mathrm{L}}-J_{\mathrm{D}}
$$

where $J_{\mathrm{L}}$ is the measured current density under illumination. Furthermore, $V_{\text {eff }}$ was calculated according to the equation

$$
V_{\text {eff }}=V_{\mathrm{o}}-V_{\mathrm{a}}
$$

where $V_{\mathrm{o}}$ is the voltage, the value of $J_{\text {ph }}$ equals zero (i.e., $J_{\mathrm{L}}=$ $\left.J_{\mathrm{D}}\right)$, and $V_{\mathrm{a}}$ is the applied bias on the devices. From Figure 6a, we can clearly see two different regimes. In the first, $J_{\mathrm{ph}}$ linearly increases with the increasing $V_{\text {eff. }}$ Then, $J_{\mathrm{ph}}$ becomes saturated at a sufficiently high value of $V_{\text {eff. }}$. We determined the value of such a photocurrent at high biases to be the saturation photocurrent density $\left(J_{\text {sat }}\right)$, which should be theoretically independent of the applied electrical field and temperature. ${ }^{27,28}$ Under the saturation conditions, all of the excitons generated in the device could be dissociated and contributed to the current owing to the high electric potential, which could overcome the exciton binding energy. Therefore, we express $J_{\text {sat }}$ as

$$
J_{\text {sat }}=\mathrm{q}_{\text {max }} L
$$

where $q$ is the electronic charge and $L$ is the thickness of the active layer. $^{27,28}$ Therefore, the maximum exciton generation rate $\left(G_{\max }\right)$ can be calculated from the measured results of $J_{\text {sat }}$. Because the value of $G_{\max }$ is relative to the maximum number of photons absorbed in the devices, it can be considered as one key parameter for evaluating the absorption ability of typical OPVs. From Figure 6a, the values of $G_{\max }$ were $3.82 \times 10^{27}$ and $4.13 \times 10^{27} \mathrm{~m}^{-3} \mathrm{~s}^{-1}$ for the device prepared without and with $\mathrm{Au}$ NPs, respectively. The noticeable increase in $G_{\max }$ for the device containing the $\mathrm{Au}$ NPs suggests an enhanced lightharvesting ability of the plasmonic device.

Furthermore, the exciton dissociation probabilities $[P(E, T)]$ were also affected by the LSPR. ${ }^{13}$ For OPVs, only a portion of the exciton can be dissociated into free carriers under normal operation conditions. Therefore, $J_{\mathrm{ph}}$ can be expressed as the following equation ${ }^{27,28}$

$$
J_{\mathrm{ph}}=q G_{\max }(E, T) L
$$

Assuming the dissociation probability is $100 \%$ under saturation conditions, the value of $P(E, T)$ under various bias conditions can be calculated from the plot of the normalized photocurrent density $\left(J_{\mathrm{ph}} / J_{\mathrm{sat}}\right)$. Figure $6 \mathrm{~b}$ displays the normalized photocurrent density. Under the short-circuit conditions $\left(V_{\mathrm{a}}=0 \mathrm{~V}\right)$, the result indicates that the probability of $P(E, T)$ was increased from 78.7 to $84.9 \%$ for the device incorporating 10\% AuNPs. We infer that excitation of the LSPR could also facilitate exciton dissociation, increasing the photocurrent of the plasmonic devices.

From the device results, we also noted that FF values were increased after the use of Au NPs. In fact, a higher exciton dissociation probability decreases the recombination rate. ${ }^{13}$ Furthermore, the device series resistance was increased slightly, suggesting that the improvement in the FF values was not due to the reduction of resistance. Therefore, we suggest that the LSPR effects increased the exciton dissociation probability, thereby increasing the FF values. ${ }^{13}$

The results of steady-state photoluminescence (PL) measurements of the P3HT:PCBM thin films prepared on various buffer layers also helped to understand the LSPR effect on the exciton generation behavior; Figure 7 shows the PL results. The peak intensity of the thin film containing Au NPs apparently became higher by approximately $60 \%$. As was noted previously, because of the good overlap between the resonance 


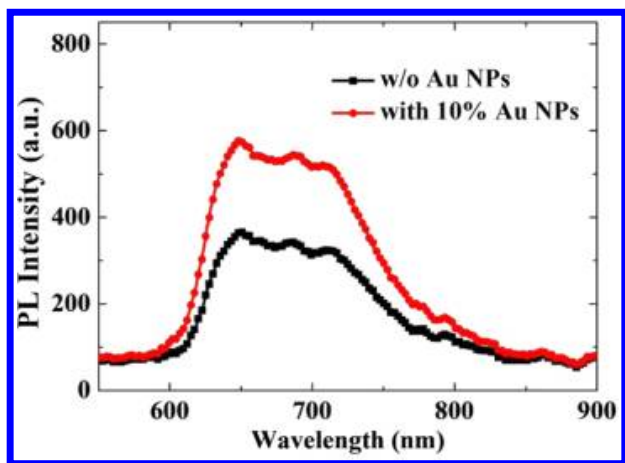

Figure 7. Photoluminescent spectra of the P3HT:PCBM thin films deposited on the $\mathrm{MoO}_{3}$ layers prepared with and without Au NPs. A $470 \mathrm{~nm}$ laser was used as the excitation source.

peak of the Au NPs and the absorption spectrum of P3HT, we attributed the increased PL intensity to the increased level of photon absorption. The enhanced PL intensity probably arises from the coupling between the plasmonic field of the Au NPs and the excited state of P3HT.

The long-term stability of devices was investigated by testing the shelf lifetime. The solar cells were stored under ambient conditions in which the relative humidity during the period of measurement was controlled at ca. $60 \%$. The test protocols could be qualified as an ISOS-D-1 experiment. ${ }^{29}$ Figure 8 shows

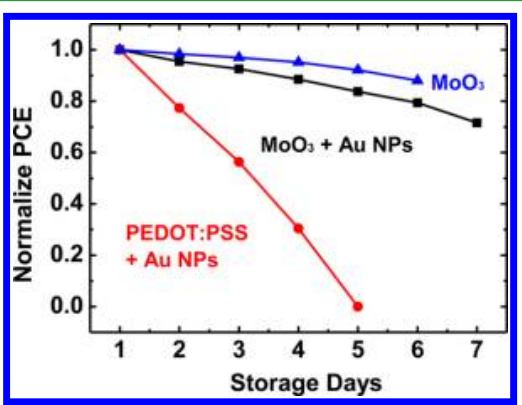

Figure 8. Normalized PCEs of devices fabricated with PEDOT:PSS or $\mathrm{MoO}_{3}$ anode buffer layers plotted as a function of time. Au NPs were incorporated into the buffer layers. The devices, which were not encapsulated, were stored under ambient conditions.

the normalized PCEs of the devices as a function of time. It can be seen that the device made of a PEDOT:PSS buffer layer degraded much more rapidly. The PCE decreased to 50\% of the initial efficiency within the first 3 days. No apparent electrical response could be measured after 5 days. In contrast, the devices fabricated with $\mathrm{MoO}_{3}$ buffer layers exhibited higher stability. The addition of Au NPs slightly affects the device stability, which is probably due to the degradation of the interface as the surface of the buffer layer becomes rougher (Figure 5). ${ }^{30}$ For the device containing $10 \% \mathrm{Au}$ NPs, however, the PCE still remained at ca. $80 \%$ of its initial value after storing the device for 7 days. The replacement of the hygroscopic PEDOT:PSS with $\mathrm{MoO}_{3}$ might reduce the diffusion rate of humidity within the devices, thus reducing the degree of oxidation of the cathode. Furthermore, unlike the acidic PEDOT:PSS, the $\mathrm{MoO}_{3}$ buffer could also avoid the corrosion of the ITO anode.

\section{CONCLUSIONS}

We have developed a simple, solution-processed method for preparing oxide buffer layer containing Au NPs for OPVs. The plasmonic effects induced by the $\mathrm{Au}$ NPs enhanced the photocurrent and PCE of the devices. More importantly, the devices fabricated with these $\mathrm{Au} \mathrm{NP} / \mathrm{MoO}_{3}$ buffer layer exhibited a longer device lifetime. We foresee that the metal/ oxide nanocomposites reported herein might be of further use in other material systems, such as low-band-gap polymers, to achieve even higher efficiencies and prolonged stability.

\section{ASSOCIATED CONTENT}

\section{Supporting Information}

$J-V$ characteristics of the devices measured in the dark, and TEM image of the nanocomposites. This material is available free of charge via the Internet at http://pubs.acs.org.

\section{AUTHOR INFORMATION}

\section{Corresponding Author}

*E-mail: fcchen@mail.nctu.edu.tw.

Notes

The authors declare no competing financial interest.

\section{ACKNOWLEDGMENTS}

We thank the National Science Council of Taiwan (grant nos. NSC 102-2221-E-009-130-MY3， NSC 102-3113-P-009-004, and NSC 101-2628-E-009-008-MY3) and the Ministry of Education of Taiwan (through the ATU program) for financial support. We also thank Prof. Michael H. Huang and Mrs. Ching-Wen Liao from National Tsing Hua University for providing the $\mathrm{Au}$ nanoparticles.

\section{REFERENCES}

(1) Yip, H. L.; Jen, A. K.-Y. Energy Environ. Sci. 2012, 5, 5994-6011.

(2) Servaites, J. D.; Ratner, M. A.; Marks, T. J. Energy Environ. Sci. 2011, 4, 4410-4422.

(3) Roes, A. L.; Alsema, E. A.; Blok, K.; Patel, M. K. Prog. Photovoltaics 2009, 17, 372-393.

(4) Li, G.; Zhu, R.; Yang, Y. Nat. Photonics 2012, 6, 153-161.

(5) Ko, D. H.; Tumbleston, J. R.; Gadisa, A.; Aryal, M.; Liu, Y.; Lopez, R.; Samulski, E. T. J. Mater. Chem. 2011, 21, 16293-16303.

(6) Park, S. H.; Roy, A.; Beaupre, S.; Cho, S.; Coates, N.; Moon, J. S.; Moses, D.; Leclerc, M.; Lee, K.; Heeger, A. J. Nat. Photonics 2009, 3, 297-302.

(7) Gan, Q.; Bartoli, F. J.; Kafafi, Z. H. Adv. Mater. 2013, 25, 23852396.

(8) Kim, J. Y.; Kim, S. H.; Lee, H.-H.; Lee, K.; Ma, W.; Gong, X.; Heeger, A. J. Adv. Mater. 2006, 18, 572-576.

(9) Chen, F. C.; Wu, J.- L.; Hung, Y. Appl. Phys. Lett. 2010, 96, 193304-1-193304-3.

(10) Wang, D. H.; Park, K. H.; Seo, J. H.; Seifter, J.; Jeon, J. H.; Kim, J. K.; Park, J. H.; Park, O. O.; Heeger, A. J. Adv. Energy Mater. 2011, 1, 766-770.

(11) Li, X.; Choy, W. C. H.; Lu, H.; Sha, W. E. I.; Ho, A. H. P. Adv. Funct. Mater. 2013, 21, 2728-2735.

(12) Chen, F. C.; Wu, J.-L.; Lee, C. L.; Hong, Y.; Kuo, C. H.; Huang, M. H. Appl. Phys. Lett. 2009, 95, 013305-1-013305-3.

(13) Wu, J. L.; Chen, F. C; Hsiao, Y. S.; Chien, F. C.; Chen, P.; Kuo, C. H.; Huang, M. H.; Hsu, C. S. ACS Nano 2011, 5, 959-967.

(14) Yang, X.; Chueh, C. C.; Li, C. Z.; Yip, H. L.; Yin, P.; Chen, H.; Chen, W. C.; Jen, A. K. Y. Adv. Energy Mater. 2013, 3, 666-673.

(15) Baek, S. W.; Noh, J.; Lee, C. H.; Kim, B.; Seo, M. K.; Lee, J. Y. Sci. Rep. 2013, 3, 1726.

(16) Hsiao, Y. S.; Charan, S.; Wu, F. Y.; Chien, F. C; Chu, C. W.; Chen, P.; Chen, F. C J. Phys. Chem. C 2012, 116, 20731-20737. 
(17) Kao, C. S.; Chen, F. C; Liao, C. W.; Huang, M. H.; Hsu, C. S. Appl. Phys. Lett. 2012, 101, 193902-1-193902-4.

(18) Jǿrengsen, M.; Norrman, K.; Krebs, F. C. Sol. Energy Mater. Sol. Cells 2008, 92, 686-714.

(19) Shrotriya, V.; Li, G.; Yao, Y.; Chu, C. W.; Yang, Y. Appl. Phys. Lett. 2006, 88, 073508-1-073508-3.

(20) Sun, Y.; Takacs, C. J.; Cowan, S. R.; Seo, J. H.; Gong, X.; Roy, A.; Heeger, A. J. Adv. Mater. 2011, 23, 2226-2230.

(21) Yang, T.; Wang, M.; Cao, Y.; Huang, F.; Huang, L.; Peng, J.; Gong, X.; Cheng, S. Z. D.; Cao, Y. Adv. Energy Mater. 2012, 2, 523527.

(22) Murase, S.; Yang, Y. Adv. Mater. 2012, 24, 2459-2462.

(23) Chang, C. C.; Wu, H. L.; Kuo, C. H.; Huang, M. H. Chem. Mater. 2008, 20, 7570-7574.

(24) Li, G.; Yao, Y.; Yang, H.; Shrotriya, V.; Yang, G.; Yang, Y. Adv. Funct. Mater. 2007, 17, 1636-1644.

(25) Chen, F. C.; Ko, C. J.; Wu, J. L.; Chen, W. C. Sol. Energy Mater. Sol. Cells 2010, 94, 2426-2430.

(26) Shrotriya, V.; Li, G.; Yao, Y.; Moriarty, T.; Emery, K.; Yang, Y. Adv. Funct. Mater. 2006, 16, 2016-2023.

(27) Mihailetchi, V. D.; Koster, L. J. A.; Hummelen, J. C.; Blom, P. W. M. Phys. Rev. Lett. 2004, 93, 216601-1-216601-4.

(28) Mihailetchi, V. D.; Xie, H. X.; de Boer, B.; Koster, L. J. A.; Blom, P. W. M. Adv. Funct. Mater. 2006, 16, 699-708.

(29) Reese, M. O.; Gevorgyan, S. A.; Jørgensen, M.; Bundgaard, E.; Kurtz, S. R.; Ginley, D. S.; Olson, D. C.; Lloyd, M. T.; Morvillo, P.; Katz, E. A.; Elschner, A.; Haillant, O.; Currier, T. R.; Shrotriya, V.; Hermenau, M.; Riede, M.; Kirov, K. R.; Trimmel, G.; Rath, T.; Inganas, O.; Zhang, F.; Andersson, M.; Tvingstedt, K.; Lira-Cantu, M.; Laird, D.; McGuiness, C.; Gowrisanker, S. (J.); Pannone, M.; Xiao, M.; Hauch, J.; Steim, R.; DeLongchamp, D. M.; R€osch, R.; Hoppe, H.; Espinosa, N.; Urbina, A.; Yaman-Uzunoglu, G.; Bonekamp, J.-B.; van Breemen, A. J. J. M.; Girotto, C.; Voroshazi, E.; Krebs, F. C. Sol. Energy Mater. Sol. Cells 2011, 95, 1253-1267.

(30) Chen, F. C.; Chien, S. C. J. Mater. Chem. 2009, 19, 6865-6869. 